\title{
Static thermodynamic quantities of quantum Heisenberg spin glasses with anisotropic interaction in applied magnetic fields
}

\author{
Y.-S. Xiong* \\ National Laboratory of Laser Technology and Physics Department of Huazhong University of Science and Technology, \\ Wuhan 430074, People's Republic of China \\ L. Yi \\ Department of Physics, Hong Kong University of Science and Technology, Clear Water Bay, Kowloon, Hong Kong \\ and Chinese Center for Advanced Science and Technology (CCAST) (World Laboratory), \\ P.O. Box 8730, Beijing 100080, People's Republic of China \\ K.-L. Yao \\ Chinese Center for Advanced Science and Technology (CCAST) (World Laboratory), \\ P.O. Box 8730, Beijing 100080, People's Republic of China \\ and International Center for Material Physics, Chinese Academy of Science, Shenyang 110015, People's Republic of China
}

(Received 13 June 1994)

\begin{abstract}
In the static replica symmetric approximation, the quantum Heisenberg spin-glass model with the infinite-ranged random Dzyaloshinskii-Moriya interaction in external magnetic fields is investigated in detail. The local susceptibility and the corresponding order parameters are calculated, which are in good agreement with those of thermofield calculations. The dependencies of entropy and specific heat on temperature are studied numerically at various magnetic fields and for different spin numbers. It is shown that the entropy is always positive and the quantum fluctuation has very strong effect on specific heat. Our results show that the typical specific heat of a spin glass for different magnetic fields exhibits crossover behavior, which has been obtained experimentally but not explained theoretically before.
\end{abstract}

\section{INTRODUCTION}

It has been found that a number of hexagonal metallic spin-glass properties are strongly influenced by various types of anisotropies. ${ }^{1-3}$ These systems may be described by a model in which, in addition to the random isotropic Heisenberg exchange interaction, a single spin uniaxial anisotropy energy $-D\left(S_{z}\right)^{2}$ is added, ${ }^{4,5}$ where $S_{z}$ is the $z$ component of the spin operator. Theoretically, uniaxial anisotropy brings about several new features. The quantum Heisenberg spin-glass model with exchange randomness and uniaxial anisotropy has been investigated by many authors, ${ }^{6-10}$ and a multiplicity of phases has been found.

Experiments on the canonical spin glass such as CuMn and AgMn in the presence of nonmagnetic impurities (for example, $\mathrm{Au}$ or $\mathrm{Pt}$ ) with strong spin-orbit coupling to the conduction electrons reveal the existence of an anisotropy which can be explained by the Dzyaloshinskii-Moriya (DM) interaction. ${ }^{11,12}$ This interaction describes the scattering of the conduction electrons of the host $(\mathrm{Cu})$ by Mn spins via the spin-orbit exchange of the nonmagnetic impurity. Theoretically, the influence of the DM interaction has been investigated in Monte Carlo simulations for classical spin-glass systems ${ }^{13-15}$ as well as in analytical studies with random DM exchange. ${ }^{16-21}$ Especially, it has been demonstrated that the specific-heattemperature curve of $\mathrm{CuMn}$ alloys at various magnetic fields exhibits crossover behavior, ${ }^{22}$ which caused a puzzling problem for theorists. In addition, the thermodynamic properties of classical spin-glass models have been studied by some authors. ${ }^{23}$ Unfortunately, these investigations neglected completely the influence of quantum features of spin operators on the thermodynamic properties of spin-glass systems. As emphasized earlier, the quantum spin glass in comparison with its classical counterpart is far from being a trivial problem due to the noncommutativity of the spin operators involved, which requires treatment by special methods. ${ }^{6,11,24-33}$ Typically, quantum mechanics manifest itself via time-dependent self-interaction and order parameters, in contrast to the classical spin-glass systems, and the dynamics becomes an inherent feature of the problem, which significantly influences the calculation of critical lines and transition points, etc. The technique employed here to deal with both randomness and quantum features was introduced by some of us $^{24}$ and has been successfully implemented in other quantum spin-glass problems. ${ }^{25}$ This theory allows one to treat both magnetic and thermodynamic problems in a unified scheme.

The purpose of this work is to continue the analysis of the Heisenberg model with exchange randomness and random DM anisotropy interaction in Refs. 24 and 25. Thermodynamic quantities are studied at various magnetic fields and for spin numbers $S=\frac{1}{2}, 1, \frac{3}{2}$, and 2 . Special attention will be paid to the analysis of the crossing 
behavior of specific-heat-temperature curves for different magnetic fields. For comparison with results by other methods, the temperature dependencies of local susceptibilities and corresponding order parameters are also investigated numerically.

\section{STATIC REPLICA SYMMETRIC FORMULATION}

The model Hamiltonian of the system is given by

$$
H=-\sum_{i j} J_{i j} \mathbf{S}_{i} \cdot \mathbf{S}_{j}-\sum_{i j} \mathbf{D}_{i j} \cdot\left(\mathbf{S}_{i} \times \mathbf{S}_{j}\right)+\mathbf{h} \cdot \sum_{i} \mathbf{S}_{i}
$$

where $\mathbf{S}=\left(S_{x}, S_{y}, S_{z}\right)$ is the quantum spin operator associated with the local moment $S$. The first two sums are over all possible distinct pairs $(i, j)$ of sites. The strengths of exchange interactions $J_{i j}$ and $\mathbf{D}_{i j}$, the latter corresponding to the DM interaction, are quenched and independently distributed with symmetric Gaussian probability distributions

$$
P\left(J_{i j}\right)=\left(\frac{N}{2 \pi J^{2}}\right)^{1 / 2} \exp \left(-\frac{N J_{i j}^{2}}{2 J^{2}}\right)
$$

and

$$
W\left(\mathbf{D}_{i j}\right)=\left(\frac{N}{2 \pi D^{2}}\right]^{3 / 2} \exp \left[-\frac{N \mathbf{D}_{i j}^{2}}{2 D^{2}}\right],
$$

respectively. As usual the scaling of the variances $J / N$ and $D / N$ ensures a sensible thermodynamic limit $N \rightarrow+\infty$. The external magnetic field $h$ is supposed to be in the direction of the $z$ axis.

The derivation of the free energy is a straightforward generalization of work of Bray and Moore. ${ }^{27}$ In order to average over the random couplings $\left\{J_{i j}\right\}$ and $\left\{\mathbf{D}_{i j}\right\}$, we apply the replica method and Matsubara imaginary-time functional-integral technique, which allows us to treat the noncommuting spin operators as $C$ numbers. The present calculation follows Ref. 24 closely; we therefore quote here only the saddle-point free energy per spin. One has

$$
\begin{aligned}
n \beta F[\mathbf{R}, \mathbf{Q}]= & {\left[\frac{J \beta}{2}\right]^{2} \int_{0}^{1} d \tau \int_{0}^{1} d \tau^{\prime}\left[\sum_{\alpha} \sum_{\mu \nu \sigma \rho} R_{\mu \nu}^{\alpha}\left(\tau, \tau^{\prime}\right) R_{\sigma \rho}^{\alpha}\left(\tau, \tau^{\prime}\right) P_{\mu \nu \sigma \rho}+\sum_{\alpha \neq \gamma} \sum_{\mu \nu \sigma \rho} Q_{\mu \nu}^{\alpha \gamma}\left(\tau, \tau^{\prime} Q_{\sigma \rho}^{\alpha \gamma}\left(\tau, \tau^{\prime}\right) P_{\mu \nu \sigma \rho}\right]\right.} \\
-\ln \operatorname{Tr} T_{\tau} \exp \left(-\beta H_{\mathrm{eff}}[\mathbf{R}, \mathbf{Q}]\right) & \\
-\beta H_{\mathrm{eff}}[\mathbf{R}, \mathbf{Q}]=\frac{1}{2}(J \beta)^{2} \int_{0}^{1} d \tau \int_{0}^{1} d \tau^{\prime} & {\left[\sum_{\alpha} \sum_{\mu \nu \sigma \rho} R_{\mu \nu}^{\alpha}\left(\tau, \tau^{\prime}\right) P_{\mu \nu \sigma \rho} S_{\sigma}^{\alpha}(\tau) S_{\rho}^{\alpha}\left(\tau^{\prime}\right)\right.} \\
& \left.+\sum_{\alpha \neq \gamma} \sum_{\mu \nu \sigma \rho} Q_{\mu \nu}^{\alpha \gamma}\left(\tau, \tau^{\prime}\right) P_{\mu \nu \sigma \rho} S_{\sigma}^{\alpha}(\tau) S_{\rho}^{\gamma}\left(\tau^{\prime}\right)\right]+\beta \int_{0}^{1} d \tau \sum_{\alpha} \mathbf{h} \cdot \mathbf{S}^{\alpha}(\tau)
\end{aligned}
$$

with

$$
\begin{aligned}
& P_{\mu v \sigma \rho}=d^{2} \delta_{\mu \nu} \delta_{\sigma \rho}+\left(1-d^{2}\right) \delta_{\mu \sigma} \delta_{v \rho} \\
& d=D / J, \text { and } \beta=1 / T,
\end{aligned}
$$

where $T_{\tau}$ denotes the "time"-ordering operator which rearranges the operators in the expansion of the exponent in the order of decreasing "time" arguments $\tau$. Functional differentiation with respect to $R_{\mu \nu}^{\alpha}\left(\tau, \tau^{\prime}\right)$ and $Q_{\mu \nu}^{\alpha \gamma}\left(\tau, \tau^{\prime}\right)$ yields

$$
\begin{aligned}
& R_{\mu \nu}^{\alpha}\left(\tau, \tau^{\prime}\right)=\left\langle T_{\tau} S_{\mu}^{\alpha}(\tau) S_{v}^{\alpha}\left(\tau^{\prime}\right)\right\rangle, \\
& Q_{\mu \nu}^{\alpha \gamma}\left(\tau, \tau^{\prime}\right)=\left\langle T_{\tau} S_{\mu}^{\alpha}(\tau) S_{\nu}^{\gamma}\left(\tau^{\prime}\right)\right\rangle,
\end{aligned}
$$

where $S_{\mu}^{\alpha}$ is a Cartesian component of $\mathbf{S}^{\alpha}$. $\alpha, \gamma=1,2, \ldots, n$ are replica indices. The angular brackets denote an average with respect to the effective Hamiltonian in (4). Nonzero values for these functions imply the dynamic spin self-interaction and spin-glass order parameter, respectively. Furthermore, within the static replica symmetric theory, the order parameters and selfinteractions are separated into longitudinal $(L)$ and transverse $(T)$ components by the decomposition

$$
\begin{aligned}
& Q_{\mu \nu}=\delta_{\mu \nu}\left[Q_{L} \delta_{\mu z}+Q_{T}\left(1-\delta_{\mu z}\right)\right], \\
& R_{\mu \nu}=\delta_{\mu \nu}\left[R_{L} \delta_{\mu z}+R_{T}\left(1-\delta_{\mu z}\right)\right]
\end{aligned}
$$

After substituting these equations into the free energy [Eqs. (3) and (4)] and applying the Hubbard-Stratonovich transformation to linearize the quadratic forms in the effective Hamiltonian, the free-energy density becomes 


$$
\begin{aligned}
\beta F[\mathbf{R}, \mathbf{Q}]=\left(\frac{J \beta}{2}\right)^{2} & \left\{R_{L}^{2}+2\left(1+d^{2}\right) R_{T}^{2}+4 d^{2} R_{L} R_{T}\right. \\
& \left.-\left[Q_{L}^{2}+2\left(1+d^{2}\right) Q_{T}^{2}+4 d^{2} Q_{L} Q_{T}\right]\right\} \\
& -\int D \mathbf{z} \ln L(\mathbf{z})
\end{aligned}
$$

$L(\mathbf{z})=\int D \mathbf{z}_{1} \Phi\left[\Omega\left(\mathbf{z}, \mathbf{z}_{1}\right)\right]$,

$\Phi\left[\Omega\left(\mathbf{z}, \mathbf{z}_{1}\right)\right]=\frac{\sinh \left[(2 S+1) \Omega\left(\mathbf{z}, \mathbf{z}_{1}\right)\right]}{\sinh \left[\Omega\left(\mathbf{z}, \mathbf{z}_{1}\right)\right]}$.

The function $\Omega\left(z, z_{1}\right)$ is given by

$$
\begin{gathered}
\Omega\left(\mathbf{z}, \mathbf{z}_{1}\right)=\frac{J \beta}{2}\left[\left(a_{1} x+a_{2} x_{1}\right)^{2}+\left(a_{1} y+a_{2} y_{1}\right)^{2}\right. \\
\left.+\left(a_{3} z+a_{4} z_{1}+\frac{h}{J}\right]^{2}\right]^{1 / 2}, \\
a_{1}=\left[d^{2} Q_{L}+\left(1+d^{2}\right) Q_{T}\right]^{1 / 2}, \\
a_{2}=\left[d^{2}\left(R_{L}-Q_{L}\right)+\left(1+d^{2}\right)\left(R_{T}-Q_{T}\right)\right]^{1 / 2}, \\
a_{3}=\left[Q_{L}+2 d^{2} Q_{T}\right]^{1 / 2}, \\
a_{4}=\left[R_{L}-Q_{L}+2 d^{2}\left(R_{T}-Q_{T}\right)\right]^{1 / 2},
\end{gathered}
$$

with the abbreviation

$$
\begin{aligned}
\int D \mathbf{z} A(\mathbf{z}) & =\langle A(\mathbf{z})\rangle_{\mathbf{z}} \\
& =\int_{-\infty}^{+\infty} \frac{d^{3} \mathbf{z}}{(2 \pi)^{3 / 2}} \exp \left(-\mathbf{z}^{2} / 2\right) A(\mathbf{z}) .
\end{aligned}
$$

The stationarity of the functional $F[\mathbf{R}, \mathbf{Q}]$ in Eq. (7) with respect to the spin self-interactions and spin-glass order parameters gives the following self-consistency equations: ${ }^{24}$

$$
\begin{aligned}
R_{L} & =\frac{1}{\left(a_{4} J \beta\right)^{2}} \int \frac{D \mathbf{z}}{L(\mathbf{z})} \int D \mathrm{z}_{1} \Phi(\Omega)\left(z_{1}^{2}-1\right) \\
R_{T} & =\frac{1}{2\left(a_{2} J \beta\right)^{2}} \int \frac{D \mathbf{z}}{L(\mathbf{z})} \int D \mathrm{z}_{1} \Phi(\Omega)\left(x_{1}^{2}+y_{1}^{2}-2\right) \\
Q_{L} & =\frac{1}{\left(a_{4} J \beta\right)^{2}} \int D \mathrm{z}\left[\frac{1}{L(\mathbf{z})} \int D \mathrm{z}_{1} \Phi(\Omega) z_{1}\right)^{2} \\
Q_{T} & =\frac{1}{\left(a_{2} J \beta\right)^{2}} \int D \mathrm{z}\left[\frac{1}{L(\mathbf{z})} \int D \mathrm{z}_{1} \Phi(\Omega) x_{1}\right]^{2}
\end{aligned}
$$

The unaveraged susceptibility is given by

$$
\chi_{\mu \nu}(\mathbf{z})=-\left.\frac{\partial m_{\mu}(\mathbf{z})}{\partial h_{\mu}}\right|_{h_{v} \rightarrow 0},
$$

while

$$
m_{\mu}(\mathbf{z})=-\left.\frac{1}{\beta L(\mathbf{z})} \frac{\partial L(\mathbf{z})}{\partial h_{\mu}}\right|_{h_{\mu} \rightarrow 0}
$$

where $h_{\Theta}(\Theta=\mu, v)$ denotes an infinitesimal applied magnetic field. Furthermore, since the rotational symmetry with respect to the $z$ axis (the direction of the fixed magnetic field) remains after the DM interaction has been averaged over, the matrix element of the local susceptibility tensor can be obtained readily as

$$
\begin{array}{r}
\chi_{\mu \nu}=\frac{1}{a_{\mu} a_{\nu} \beta J^{2}} \int D \mathbf{z}\left(\frac{1}{L(\mathbf{z})} \int D \mathbf{z}_{1} \Phi(\Omega)\left(\mu_{1} v_{1}-\delta_{\mu \nu}\right)\right. \\
-\frac{1}{L^{2}(\mathbf{z})} \int D \mathbf{z}_{1} \Phi(\Omega) \mu_{1} \\
\left.\times \int D \mathbf{z}_{1} \Phi(\Omega) v_{1}\right),
\end{array}
$$

where $a_{x}=a_{y}=a_{2}$ and $a_{z}=a_{4}$.

One of the advantages in the present approximation is to calculate directly the thermodynamic quantities. From the free energy in (7), therefore, we will focus here on the entropy and the specific heat. Using the thermodynamic formula, the entropy of the system with fixed volume $V$ is given by

$$
\begin{gathered}
S_{V}=\left(\frac{J \beta}{2}\right]^{2}\left\{R_{L}^{2}+2\left(1+d^{2}\right) R_{T}^{2}+4 d^{2} R_{L} R_{T}\right. \\
\left.-\left[Q_{L}^{2}+2\left(1+d^{2}\right) Q_{T}^{2}+4 d^{2} Q_{L} Q_{T}\right]\right\} \\
+\int D \mathbf{z} \ln L(\mathbf{z})-\int \frac{D \mathbf{z}}{L(\mathbf{z})} \int D \mathbf{z}_{1} \Omega \frac{\partial \Phi}{\partial \Omega}
\end{gathered}
$$

and the corresponding specific heat is

$$
\begin{aligned}
C_{V}=-\frac{(J \beta)^{2}}{2}\{ & R_{L}^{2}+2\left(1+d^{2}\right) R_{T}^{2}+4 d^{2} R_{L} R_{T} \\
& \left.-\left[Q_{L}^{2}+2\left(1+d^{2}\right) Q_{T}^{2}+4 d^{2} Q_{L} Q_{T}\right]\right\} \\
& +\int \frac{D \mathbf{z}}{L(\mathbf{z})} \int D \mathbf{z}_{1} \Omega^{2} \frac{\partial^{2} \Phi(\Omega)}{\partial^{2} \Omega} \\
& \left.-\int \frac{D \mathbf{z}}{L^{2}(\mathbf{z})} \iint D \mathbf{z}_{1} \Omega^{2} \frac{\partial \Phi(\Omega)}{\partial \Omega}\right]^{2}
\end{aligned}
$$

Since both the entropy and the specific heat are functions of order parameters $\mathbf{R}$ and $\mathbf{Q}$ (which depend on temperature), they must be determined self-consistently by Eqs. (10)-(13). The corresponding numerical calculations will be carried out in the next section. 


\section{NUMERICAL SOLUTIONS AND DISCUSSION}

In order to avoid complications caused by mixing of various longitudinal and transverse modes in selfconsistency equations, we restrict our considerations to weak anisotropy $d=0.2$. However, it turns out that in this restricted case a great richness of thermodynamic features already occurs.

Figure 1 shows that spin self-interactions and spinglass order parameters change with temperature for different magnetic fields for fixed reduced anistropy $d=0.2$ and spin number $S=\frac{1}{2}$. Obviously, the spin-glass order parameters and corresponding self-interactions tend to coincide as temperature decreases. The longitudinal components of spin self-interaction and spin-glass order parameter $R_{L}, Q_{L}$ increase with magnetic field. Longitudinal spin-glass order parameters $Q_{L}$ are always nonzero in external magnetic fields. There exists crossover behavior for transverse spin-glass order parameters $Q_{T}$ in different magnetic fields, while the transverse spin self-interaction $R_{T}$ decreases as the magnetic field increases. Figure 2 gives the longitudinal and transverse susceptibility-temperature curves for the same conditions as in Fig. 1. It is found that the longitudinal and transverse susceptibilities are mixed up strongly due to the DM anisotropy. The result is in qualitative agreement with that of the thermofield dynamics calculations by Kopeć and Büttner. ${ }^{21}$

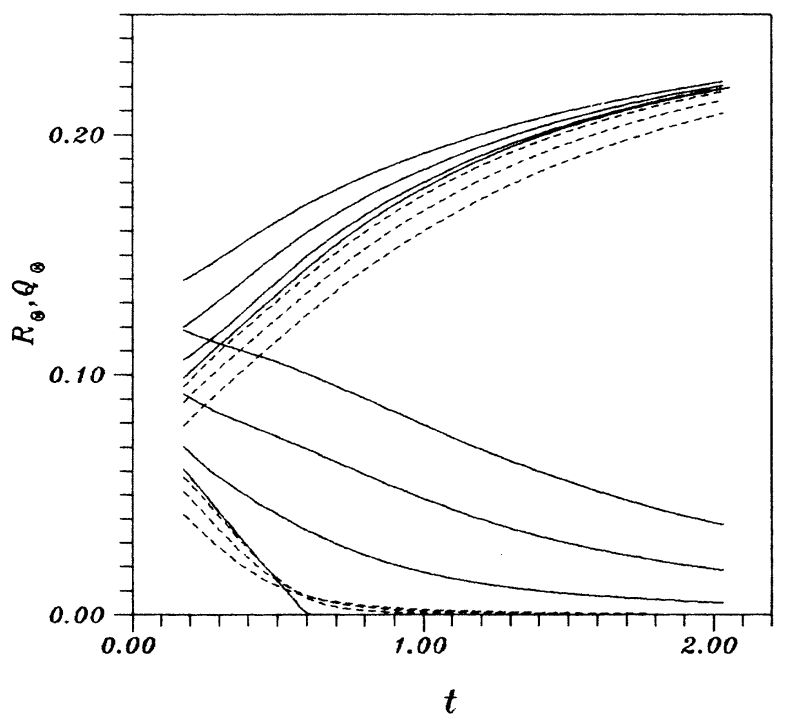

FIG. 1. Temperature dependence of the longitudinal $\left(R_{L}\right.$ and $Q_{L}$, solids lines) and transverse ( $R_{T}$ and $Q_{T}$, dashed lines) spinglass order parameters and spin self-interactions for different reduced magnetic field $\tilde{h}=h / J$, where the upper lines refer to $R_{\Theta}$ and the lower lines refer to $Q_{\Theta} . \widetilde{h}=0.0,0.1,0.2$, and 0.3 correspond to longitudinal components from top to bottom and transverse components from bottom to top, $S=\frac{1}{2}$, and $d=0.2$ $(t=4 T / J)$.

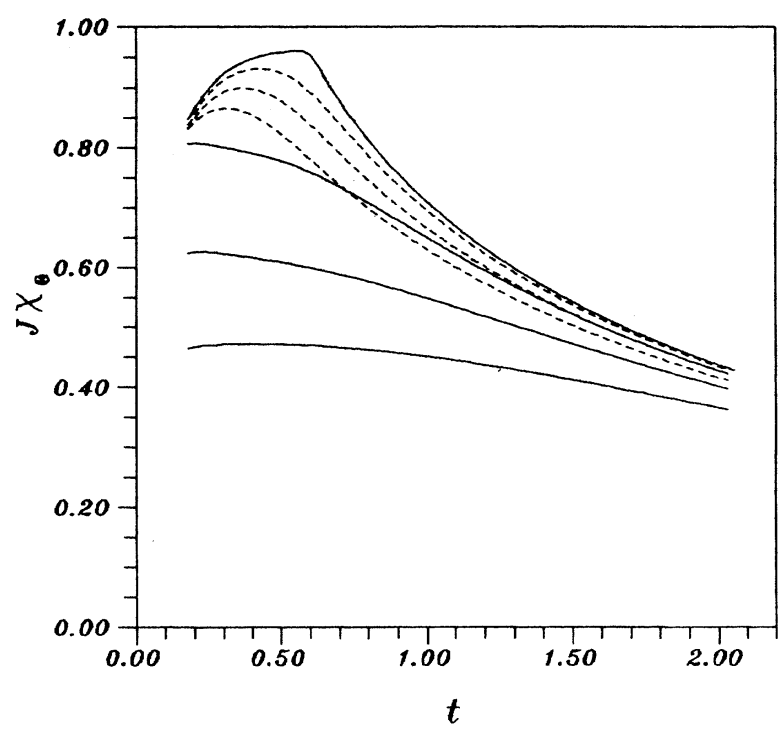

FIG. 2. Local susceptibility variation with temperature $(t=4 T / J)$ for the same conditions as in Fig. 1. The longitudinal (solid lines) and the transverse (dashed lines) components correspond to the reduced magnetic fields $\widetilde{h}=0.0,0.1,0.2$, and 0.3 from top to bottom, respectively.

The entropy-temperature curves are plotted in Fig. 3. It has been demonstrated that the entropy has weak dependence on external magnetic fields. It is easy to see in Fig. 3(c) that reducing the spin number $S$ and thereby increasing the effect of quantum fluctuations results in a strong reduction of the entropy. In addition, it is obvious that the entropy is positive at zero temperature. We argue that, since DM ansiotropy suppresses quantum fluctuation at low temperature, the replica symmetric solutions become stable. ${ }^{24}$

Figure 4 shows the temperature dependence of specific heat. It is found that the cusps of specific-heat curves are smoothed down by the external magnetic field. Especially, the specific-heat curves for different magnetic fields [see Figs. 4(a) and (b)] exhibit crossover behavior, which has been observed by Brodale et al. ${ }^{22}$ in experiments on pure CuMn samples. We found that this feature appears even if there exists a weak random DM interaction. Additionally, we can also see that the broad peak of specific heat in Fig. 4(c) moves towards high temperature and the specific heat becomes larger as the spin number increases.

In principle, for the quantum spin-glass problem an exact calculation of the thermodynamic quantities, such as specific heat and entropy, requires precise knowledge of the time dependence of the spin self-interaction involved. Therefore, this means that calculation of the exact thermodynamic quantities will depend on the detailed time dependence of $R(\tau)$ in the present Matsubara approach with the replica method. It seems that the complexity of the problem prevents an analytically tractable approach. 
However, as was pointed out earlier, ${ }^{24}$ the static approximations will give rather good results if the temperature is not too low. On the other hand, compared with the results of the thermofield dynamics ${ }^{21}$ approximation, the present calculations are in basic agreement. We argue that the reason may be that both the static and instan- taneous approximations neglect the dependence of spin self-interaction on the time.

In summary, the quantum Heinsenberg spin-glass model with DM anisotrpy is studied. The local susceptibility and the corresponding order parameters are calculated, and are in good agreement with those of thermofield dy-

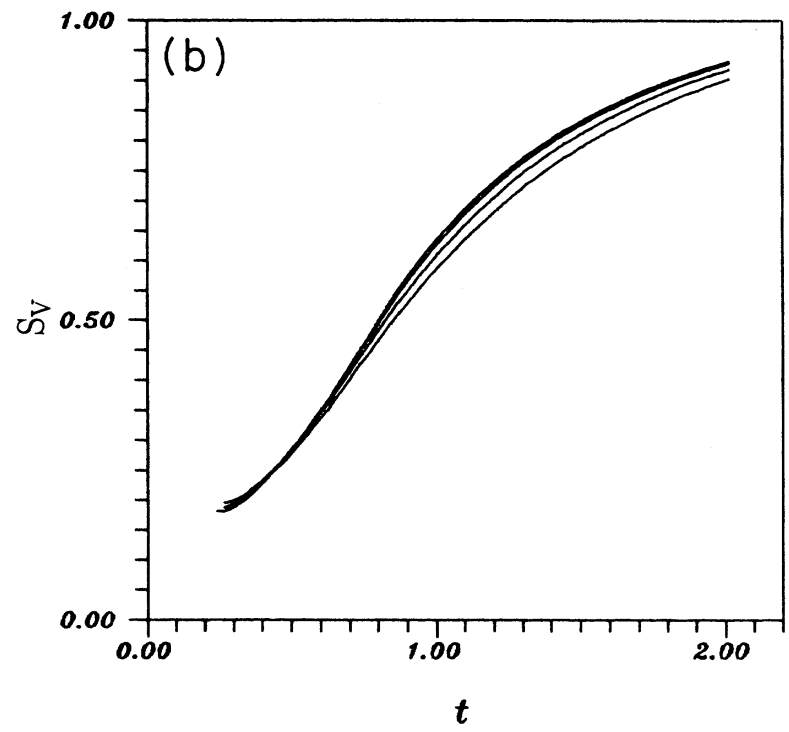

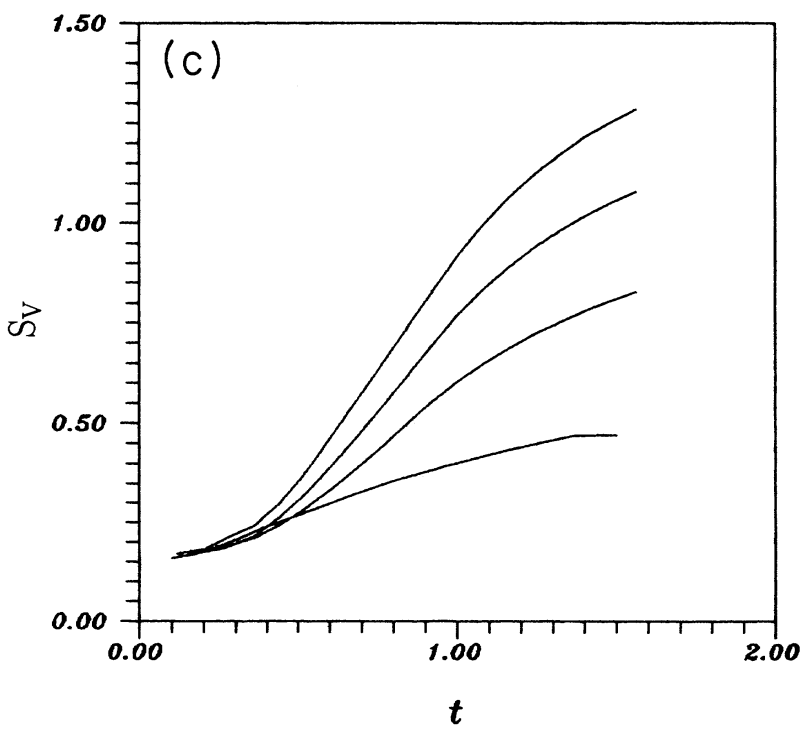

FIG. 3. (a) Entropy-temperature curves at reduced external magnetic fields $\widetilde{h}=0.0,0.1,0.2$, and 0.3 (from bottom to top) for $S=\frac{1}{2}$ and $d=0.2$, where $t=4 T / J$. (b) Entropy-temperature curves at reduced external magnetic fields $\widetilde{h}=0.0,0.1,0.2$, and 0.3 (from bottom to top) for $S=1$ and $d=0.2$, where $t=3 T /(2 J)$. (c) Entropy-temperature curves for different spin numbers $S=\frac{1}{2}, 1, \frac{3}{2}$, and 2 (from bottom to top) at $d=0.3$ and $\tilde{h}=0.1$, where $t=3 T / S(S+1) J$. 



FIG. 4. (a) Specific-heat-temperature curves for the same conditions as in Fig. 3(a). The curves from top to bottom are for $\tilde{h}=0.0,0.1,0.2$, and 0.3 , respectively, where $t=4 T / J$. (b) Specific-heat-temperature curves for the same conditions as in Fig. 3(b). The curves from top to bottom are for $\widetilde{h}=0.0,0.1,0.2$, and 0.3 , respectively. The inset shows the specific heat observed by Brodale et al. in Ref. 22, where $t=3 T /(2 J)$. (c) Specific-heat-temperature curves for the same conditions as in Fig. 3(c). The curves from bottom to top are for $S=\frac{1}{2}, 1, \frac{3}{2}$, and 2, respectively, where $t=3 T / S(S+1) J$.

namics. Due to the DM interaction, which suppressed the quantum fluctuation at low temperature, the entropy is found to be positive definite. It is shown theoretically that the specific heat of spin glasses at various magnetic fields exhibits the typical crossover behavior found by Brodale et al. ${ }^{22}$ in experiment.

\section{ACKNOWLEDGMENTS}

Two of the authors (L. Yi and K.-L. Yao) are grateful to Professor K. D. Usadel for many stimulating discussions. The project is supported by the National Natural Science Foundation of China. 
"Mailing address: Department of Physics, Huazhong University of Science and Technology, Wuhan 430074, People's Republic of China.

${ }^{1}$ H. Albrecht, E. F. Wassermann, F. T. Hedgecock, and P. Monod, Phys. Rev. Lett. 48, 891 (1982).

${ }^{2}$ A. Fert, P. Pureur, F. Hippert, K. Baberschke, and F. Bruss, Phys. Rev. B 26, 5300 (1982).

${ }^{3}$ S. Murayama, K. Yokosawa, Y. Miyako, and E. F. Wassermann, Phys. Rev. Lett. 57, 1785 (1986).

${ }^{4}$ K. D. Usadel, K. Bien, and H.-J. Sommers, Phys. Rev. B 27 , 6957 (1983).

${ }^{5}$ G. Brieskorn and K. D. Usadel, J. Phys. C 19, 3413 (1986).

${ }^{6}$ D. M. Cragg and D. Sherrington, Phys. Rev. Lett. 16, 1190 (1982).

${ }^{7}$ S. A. Roberts and A. J. Bray, J. Phys. C 15, L527 (1982).

${ }^{8}$ D. J. Elderfield and D. Sherrington, J. Phys. A 15, L437 (1982).

${ }^{9}$ H.-J. Sommers and K. D. Usadel, Z. Phys. B 47, 63 (1982).

${ }^{10}$ T. K. Kopeć, G. Büttner, and K. D. Usadel, Phys. Rev. B 41, 9221 (1990).

${ }^{11}$ I. Dzyaloshinskii, J. Phys. Chem. Solids 4, 241 (1958); T. Moriya, Phys. Rev. Lett. 4, 228 (1960).

${ }^{12}$ A. Fert and P. M. Levy, Phys. Rev. Lett. 44, 1538 (1980); P. M. Levy and A. Fert, Phys. Rev. B 23, 4667 (1981).

${ }^{13}$ S. Schultz, E. M. Gullikson, D. R. Fredkin, and M. Towar, Phys. Rev. Lett. 45, 1508 (1980).

${ }^{14}$ L. R. Walker and L. M. Walstedt, Phys. Rev. Lett. 47, 1624 (1981); J. Magn. Magn. Mater. 31-34, 1289 (1983).

${ }^{15}$ C. Dasgupta and K.-L. Yao, Phys. Rev. B 28, 4071 (1984).

${ }^{16}$ G. G. Kenning, D. Chu, and R. Orbach, Phys. Rev. Lett. 66,
2923 (1991).

${ }^{17}$ G. Kotliar and H. Sompolinsky, Phys. Rev. Lett. 53, 1751 (1984).

${ }^{18}$ K. H. Fisher, Z. Phys. B 60, 151 (1985).

${ }^{19}$ P. M. Goldbart, J. Phys. C 18, 2183 (1985).

${ }^{20} \mathrm{G}$. Büttner and K. D. Usadel, Europhys. Lett. 14, 165 (1991).

${ }^{21}$ T. K. Kopeć and G. Büttner, Phys. Rev. B 43, 10853 (1991).

${ }^{22}$ G. E. Brodale, R. A. Fisher, W. E. Fogle, N. E. Phillips, and J. van Curen, J. Magn. Magn. Mater. 31-34, 1331 (1983).

${ }^{23}$ S. Kirkpatrick and D. Sherrington, Phys. Rev. B 17, 4384 (1978).

${ }^{24}$ L. Yi, G. Büttner, K. D. Usadel, and K.-L. Yao, Phys. Rev. B 47, 254 (1993); L. Yi and K.-L. Yao, Acta Phys. Sin. 42, 488; 42, 992 (1993).

${ }^{25}$ Y. S. Xiong, L. Yi, K.-L. Yao, and Z.-G. Li, Chin. Phys. Lett. 11, 307 (1994).

${ }^{26}$ H. J. Sommers, J. Magn. Magn. Mater. 22, 267 (1980).

${ }^{27}$ A. J. Bray and M. A. Moore, J. Phys. C 13, L655 (1980).

${ }^{28} \mathrm{~K}$. Walasek and K. Lukierskia-Walasek, Phys. Rev. B 34, 4962 (1986).

${ }^{29}$ Y. Yamamoto, J. Phys. C 21, 4377 (1988).

${ }^{30}$ D. Thirumalai, Q. Li, and T. B. Kirkpatrick, J. Phys. A 22, 3339 (1991).

${ }^{31}$ Y. Y. Goldschmidt and P.-Y. Lai, Phys. Rev. B 43, 11434 (1991).

${ }^{32}$ K. D. Usadel, Solid State Commun. 58, 629 (1986).

${ }^{33}$ N. D. Mackenzie and A. P. Young, Phys. Rev. Lett. 49, 301 (1982). 